\title{
ON DISTRIBUTIONS OF CERTAIN WIENER FUNCTIONALS ${ }^{(1)}$
}

\author{
BY \\ M. KAC
}

1. Introduction. The purpose of this paper is to present a unified approach toward the problem of calculating the distribution function of the Wiener functional

$$
\int_{0}^{t} V(x(\tau)) d \tau
$$

where $x(t)$ is an element of Wiener's space $(0 \leqq t<\infty)$ and $V(x)$ is subject to certain restrictions. The most severe of these restrictions is that $V(x)$ be nonnegative, or somewhat more generally, bounded from below.

Our principal result is the following: if $\sigma(\alpha ; t)$ is the distribution function of (1.1), then

$$
\int_{0}^{\infty} \int_{0}^{\infty} \exp (-u \alpha-s t) d_{\alpha} \sigma(\alpha ; t) d t=\int_{-\infty}^{\infty} \psi(x) d x,
$$

where $\psi(x)$ is the fundamental solution (Green's function) of the differential equation

$$
\frac{1}{2} \frac{d^{2} \psi}{d x^{2}}-(s+u V(x)) \psi=0, \quad x \neq 0
$$

subject to the conditions

$$
\begin{aligned}
\psi(x) & \rightarrow 0, & x \rightarrow \pm \infty, \\
\psi^{\prime}(x) \mid & <M, & x \neq 0, \\
\psi^{\prime}(+0)-\psi^{\prime}(-0) & =-2 &
\end{aligned}
$$

The existence and uniqueness of such a fundamental solution are parts of the assertion.

The differential equation (1.3) is quite similar to the equation of Schrödinger in quantum mechanics. In fact, the results of this paper were strongly influenced by the derivation of Schrödinger's equation which we found in a hitherto unpublished Princeton Thesis of R. P. Feynman. The principal motivation behind the investigation of the distribution functions of functionals (1.1) is the following: Let $X_{1}, X_{2}, \cdots$ be identically distributed random variables each having mean 0 and variance 1 . Let furthermore

Presented to the Society, October 25, 1947; received by the editors October 17, 1947.

(1) This investigation was begun while the author was a John Simon Guggenheim Memorial Fellow. It was completed under an ONR contract. 


$$
s_{k}=X_{1}+\cdots+X_{k}
$$

and consider the random variable

$$
\frac{1}{n} \sum_{k \leqq n t} V\left(\frac{s_{k}}{n^{1 / 2}}\right)
$$

Then, under suitable restrictions on $V$, and in certain cases some further restrictions on the $X$ 's, the limiting distribution of $(1.4)$ is $\sigma(\alpha ; t)$. (For the special case $V(x)=|x|$ see $[2](2))$. Also certain generalizations of the results of this paper to processes $x(t)$ other than those of Wiener may prove to be useful in the theory of random noise.

2. Preliminary considerations. Let $x(t), x(0)=0,0 \leqq t<\infty$, be elements of the Wiener space and let $V(x)$ be a piecewise continuous, non-negative function defined for $-\infty<x<\infty$. Consider the functional

$$
\int_{0}^{t} V(x(\tau)) d \tau
$$

This functional is measurable and hence it has a distribution function which we denote by $\sigma(\alpha ; t)$. In other words,

$$
\text { Prob. }\left\{\int_{0}^{t} V(x(\tau)) d \tau<\alpha\right\}=\sigma(\alpha ; t) .
$$

It also follows that for $u>0$,

$$
E\left\{\exp \left(-u \int_{0}^{t} V(x(\tau)) d \tau\right)\right\}=\int_{0}^{\infty} \exp (-u \alpha) d_{\alpha} \sigma(\alpha ; t)=f(u ; t) .
$$

We see that $f(u ; t)$ is a bounded nonincreasing function of $t$ and consequently the real Laplace transform

$$
\int_{0}^{\infty} \exp (-s t) f(u ; t) d t=g(u ; s),
$$

exists.

Now, let $n$ be an integer and consider the sum

$$
\frac{1}{n} \sum_{k \leqq n t} V\left(x\left(\frac{k}{n}\right)\right) .
$$

As $n \rightarrow \infty$, the distribution function of this sum approaches the distribution function $\sigma(\alpha ; t)$ of the integral (2.1) at each continuity point of the latter.

Let $G_{1}, G_{2}, \cdots$ be independent, normally distributed random variables each having mean zero and variance 1 . From the definition of the Wiener

(2) Numbers in brackets refer to the bibliography at the end of the paper. 
measure it follows that the distribution function of $(2.5)$ is the same as that of

$$
\frac{1}{n} \sum_{k \leqq n t} V\left(\frac{G_{1}+\cdots+G_{k}}{n^{1 / 2}}\right) \text {. }
$$

Thus the distribution function of (2.6) approaches, as $n \rightarrow \infty, \sigma(\alpha ; t)$ at each continuity point of the latter. Consequently,

$$
f_{n}(u ; t)=E\left\{\exp \left(-\frac{u}{n} \sum_{k \leqq n t} V\left(\frac{G_{1}+\cdots+G_{k}}{n^{1 / 2}}\right)\right)\right\} \rightarrow f(u ; t) .
$$

Since $f_{n}(u ; t)$ and $f(u ; t)$ are non-negative and bounded by 1 we also have that

$$
\int_{0}^{\infty} \exp (-s t) f_{n}(u ; t) d t \rightarrow \int_{0}^{\infty} \exp (-s t) f(u ; t) d t, \quad s>0 .
$$

Noticing that for

$$
k / n \leqq t<(k+1) / n
$$

one has

$$
f_{n}(u ; t)=f_{n}(u ; k / n)
$$

we obtain

$$
\begin{aligned}
\int_{0}^{\infty} f_{n}(u ; t) \exp (-s t) d t & =\sum_{k=0}^{\infty} f_{n}\left(u ; \frac{k}{n}\right) \int_{k / n}^{(k+1) / n} \exp (-s t) d t \\
& =\frac{1-\exp (-s / n)}{s} \sum_{k=0}^{\infty} f_{n}\left(u ; \frac{k}{n}\right) \exp \left(-\frac{k}{n} s\right) .
\end{aligned}
$$

Thus

$$
\lim _{n \rightarrow \infty} \frac{1}{n} \sum_{k=1}^{\infty} f_{n}\left(u ; \frac{k}{n}\right) \exp \left(-\frac{k-1}{n} s\right)=g(u ; s), \quad s>0 .
$$

In many instances it is possible to show that $\sigma(\alpha ; t)$ is not only the limiting distribution function of (2.6) but also of

$$
\frac{1}{n} \sum_{k \leqq n t} V\left(\frac{X_{1}+\cdots+X_{k}}{n^{1 / 2}}\right)
$$

where the $X$ 's are quite general independent, identically distributed, random variables each having mean 0 and variance 1 . In [1] and [3] such proofs were carried out for $V(x)=x^{2}, V(x)=|x|$ and $V(x)=(1+\operatorname{sign} x) / 2$. The method of proof is applicable to a wide class of functions but it may be necessary to put further restrictions on the $X^{\prime}$ s. 
For instance, if $V(x)=x^{2 m}, m>1$, the method of proof will require the assumption that the $2 m$ th moment of $X_{j}$ be finite [4].

Instead of trying to state the rather cumbersome conditions under which one can prove (by the method of [1] and [3]) that the distribution function of (2.10) approaches $\sigma(\alpha ; t)$ we shall simply assume that $V(x)$ is such that the proof can be carried out. It is, of course, understood that whenever necessary we shall assume that some higher moments of $X_{j}$ are finite.

Given such a $V(x)$, we take for $X_{j}$ 's the random variables for which

$$
\text { Prob. }\left\{X_{j}=1\right\}=\text { Prob. }\left\{X_{j}=-1\right\}=1 / 2 \text {. }
$$

Setting

$$
h_{n}(u ; t)=E\left\{\exp \left(-\frac{u}{n} \sum_{k \leqq n t} V\left(\frac{X_{1}+\cdots+X_{k}}{n^{1 / 2}}\right)\right)\right\}
$$

we obtain, as before,

$$
\lim _{n \rightarrow \infty} \frac{1}{n} \sum_{k=1}^{\infty} h_{n}\left(u ; \frac{k}{n}\right) \exp \left(-\frac{k-1}{n} s\right)=g(u ; s), \quad s>0 .
$$

3. The difference equation. Let $\tau=1 / n, \Delta=1 / n^{1 / 2}$ and, for $m$ an integer,

$$
\rho(m)=\left\{\begin{array}{cl}
1 / 2 & \text { if } m=1,-1, \\
0 & \text { otherwise. }
\end{array}\right.
$$

Then (suppressing $n$ and $u$ in some places, for the sake of brevity)

$$
\begin{aligned}
h(k \tau) & =h_{n}(u ; k / n) \\
& =\sum_{m_{1}, \cdots, m_{k}} \exp \left(-u \tau \sum_{j=1}^{k} V\left(m_{j} \Delta\right)\right) \rho\left(m_{1}\right) \rho\left(m_{2}-m_{1}\right) \cdots \rho\left(m_{k}-m_{k-1}\right)\left({ }^{3}\right) .
\end{aligned}
$$

Set

$$
\phi\left(m_{k} \Delta, k \tau\right)=\sum_{m_{1}, \cdots, m_{k-1}} \exp \left(-u \tau \sum_{1}^{k} V\left(m_{j} \Delta\right)\right) \rho\left(m_{1}\right) \cdots \rho\left(m_{k}-m_{k-1}\right)
$$

and note that

$$
\phi\left(m_{k} \Delta, k \tau\right)=\exp \left(-u \tau V\left(m_{k} \Delta\right)\right) \sum_{m_{k-1}} \phi\left(m_{k-1} \Delta,(k-1) \tau\right) \rho\left(m_{k}-m_{k-1}\right) .
$$

Writing $m$ instead of $m_{k}$ and recalling the definition of $\rho(m)$ we get

$$
\begin{array}{rlrl}
\phi(m \Delta, k \tau)= & \exp (-u \tau V(m \Delta))\{\phi((m-1) \Delta,(k-1) \tau) & \\
& +\phi((m+1) \Delta,(k-1) \tau)\} / 2, \quad k>1,
\end{array}
$$

(3) Unless otherwise stated summations are understood to be extended from $-\infty$ to $\infty$. 


$$
\phi(m \Delta, \tau)=\exp (-u \tau V(m \Delta)) \rho(m) .
$$

Now,

$$
\begin{aligned}
\sum_{k=1}^{\infty} \phi(m \Delta, k \tau) \exp (-(k-1) \tau s) & \\
=\exp (-u \tau V(m \Delta)) & \left\{\rho(m)+\frac{1}{2} \exp (-s \tau) \sum_{k=2}^{\infty}[\phi((m-1) \Delta,(k-1) \tau)\right. \\
& \quad+\phi((m+1) \Delta,(k-1) \tau)] \exp (-(k-2) s \tau)\}
\end{aligned}
$$

and setting

$$
\chi(m \Delta ; s)=\tau \sum_{k=1}^{\infty} \phi(m \Delta, k \tau) \exp (-(k-1) s \tau)
$$

we obtain after a few simple transformations

$$
\frac{\chi_{m-1}-2 \chi_{m}+\chi_{m+1}}{2 \tau}-\frac{\exp \left\{\left(s+u V_{m}\right) \tau\right\}-1}{\tau} \chi_{m}=-\rho(m) \exp (s \tau) .
$$

Here we use the abbreviations

$$
V_{m}=V(m \Delta), \quad \chi_{m}=\chi(m \Delta ; s) .
$$

Introducing the function

$$
\psi_{m}=\psi(m \Delta)=\psi(m \Delta ; s)=\chi(m \Delta ; s) / \Delta
$$

and noticing that $\Delta^{2}=\tau$ we have

$$
\text { (3.6) } \frac{1}{2} \frac{\psi_{m-1}-2 \psi_{m}+\psi_{m+1}}{\Delta^{2}}-\frac{\exp \left\{\left(s+u V_{m}\right) \tau\right\}-1}{\tau} \psi_{m}=-\frac{\rho(m)}{\Delta} \exp (s \tau) \text {. }
$$

Furthermore,

$$
\frac{1}{n} \sum_{k=1} h_{n}\left(u ; \frac{k}{n}\right) \exp \left(-\frac{k-1}{n} s\right)=\Delta \sum_{m=-\infty}^{\infty} \psi_{m} .
$$

4. Passage to the limit. On purely intuitive grounds we see that the difference equation (3.6) is related to the differential equation

$$
\frac{1}{2} \frac{d^{2} \psi}{d x^{2}}-(s+u V(x)) \psi=0, \quad x \neq 0 .
$$

Furthermore, since the right-hand side of (3.6) behaves singularly near $0(m=1,-1)$ we can suspect that $\psi_{m}=\psi(m \Delta)$ will, in the limit $\Delta \rightarrow 0$, be related to a solution of (4.1) which exhibits a singularity at $x=0$. In fact, we are going to prove that 


$$
g(u ; s)=\lim _{\Delta \rightarrow 0} \Delta \sum_{m} \psi_{m}=\int_{-\infty}^{\infty} \psi(x) d x,
$$

where $\psi(x)=\psi(x ; s)$ is the Green's function (fundamental solution) of (4.1) satisfying the boundary conditions

$$
\psi(x) \rightarrow 0, \quad x \rightarrow \pm \infty
$$

and which is singular at $x=0$. The proof of this statement will be based on the method of Courant, Friedrichs and Lewy [5].

We first notice that (see (3.2))

$$
\begin{aligned}
\phi(m \Delta, k \tau) & \leqq \sum_{m_{1}, \cdots, m_{k-1}} \rho\left(m_{1}\right) \rho\left(m_{2}-m_{1}\right) \cdots \rho\left(m-m_{k-1}\right) \\
& =\text { Prob. }\left\{X_{1}+X_{2}+\cdots+X_{k}=m\right\} \\
& =\frac{1}{2 \pi} \int_{0}^{2 \pi} \exp (-i m \xi) \cos ^{k} \xi d \xi .
\end{aligned}
$$

Thus

$$
\begin{aligned}
\psi_{m}=\frac{\chi_{m}}{\Delta} & =\frac{\tau}{\Delta} \sum_{k=1}^{\infty} \phi(m \Delta, k \tau) \exp \{-(k-1) s \tau\} \\
& \leqq \frac{\tau}{2 \pi \Delta} \int_{0}^{2 \pi} \frac{\cos m \xi \cos \xi}{1-\cos \xi \exp (-s \tau)} d \xi \\
& \leqq \frac{\tau}{2 \pi \Delta} \int_{0}^{2 \pi} \frac{d \xi}{1-\cos \xi \exp (-s \tau)}=\frac{\tau}{\Delta}(1-\exp (-2 s \tau))^{-1 / 2} .
\end{aligned}
$$

Since, as $\tau \rightarrow 0\left(\Delta^{2}=\tau\right)$, we have

$$
\frac{\tau}{\Delta}(1-\exp (-2 s \tau))^{-1 / 2} \rightarrow(2 s)^{-1 / 2},
$$

we see that

$$
\psi_{m}<C(s)
$$

where $C(s)$ depends only on $s$.

From the inequality

$$
0 \leqq \psi_{m}<\frac{\tau}{2 \pi \Delta} \int_{0}^{2 \pi} \frac{\cos m \xi \cos \xi}{1-\cos \xi \exp (-s \tau)} d \xi
$$

we infer that for a fixed $\tau$ (and hence $\Delta$ ) we have

$$
\psi_{m} \rightarrow 0 \text { as } m \rightarrow \pm \infty \text {. }
$$

Multiplying both sides of (3.6) by $\Delta \psi_{m}$ and summing over $m$, we obtain 


$$
\begin{aligned}
\frac{\Delta}{2} \sum_{m}\left(\frac{\psi_{m+1}-\psi_{m}}{\Delta}\right)^{2}+\Delta \sum_{m} \frac{\exp \left\{\left(s+u V_{m}\right) \tau\right\}}{\tau}-1 & \psi_{m}^{2} \\
& =\frac{\exp (s \tau)}{2}\left(\psi_{1}+\psi_{-1}\right),
\end{aligned}
$$

after having noticed that (4.4) implies the identity

$$
\sum_{m}\left(\psi_{m-1}-2 \psi_{m}+\psi_{m-1}\right) \psi_{m}=-\sum_{m}\left(\psi_{m+1}-\psi_{m}\right)^{2} .
$$

From (4.5) and (4.3) it follows that

$$
\Delta \sum_{m}\left(\frac{\psi_{m+1}-\psi_{m}}{\Delta}\right)^{2}<2 C(s) \exp (s \tau)<D(s),
$$

where $D(s)$ again depends on $s$ alone. Consider now the functions $\psi_{\Delta}(x)$ whose graphs are polygonal lines joining the points $\left(m \Delta, \psi_{m}\right)$. These functions are uniformly bounded (see (4.3)), and, moreover, they are equicontinuous. To see this we write

$$
\begin{aligned}
|\psi(p \Delta)-\psi(q \Delta)| & =\left|\sum_{m=q}^{p-1}\left(\psi_{m+1}-\psi_{m}\right)\right| \\
& \leqq(p-q)^{1 / 2}\left[\sum_{m}\left(\psi_{m+1}-\psi_{m}\right)^{2}\right]^{1 / 2} \\
& <(D(s))^{1 / 2}(p \Delta-q \Delta)^{1 / 2} .
\end{aligned}
$$

Thus one can chose a subsequence, from the sequence $\psi_{\Delta}(x)$, which converges uniformly (in every finite interval) to a continuous function $\psi(x)$. For $m \neq 1$, -1 we have (see 3.6 )),

$$
\frac{1}{2} \frac{\psi_{m-1}-2 \psi_{m}+\psi_{m+1}}{\Delta^{2}}=\frac{\exp \left\{\left(s+u V_{m}\right) \tau\right\}-1}{\tau} \psi_{m}
$$

and consequently, if $l$ and $n$ are integers $(l<n)$ both less than -1 or both greater than 1, we get by double summation

(4.6) $\frac{\psi_{n}}{2}-\frac{\psi_{l}}{2}-\frac{(n-l) \Delta}{2} \frac{\psi_{l}-\psi_{l-1}}{\Delta}=\Delta^{2} \sum_{j=l}^{n-1} \sum_{m=l}^{j} \frac{\exp \left\{\tau\left(s+u V_{m}\right)\right\}-1}{\tau} \psi_{m}$.

If $\Delta \rightarrow 0$ (through the chosen subsequence) and $n \Delta \rightarrow x, l \Delta \rightarrow x_{0}$ we see that the right-hand side of (4.6) approaches

$$
\int_{x_{0}}^{x} \int_{x_{0}}^{\xi}(s+u V(x)) \psi(x) d x d \xi
$$

Since $\psi_{n} \rightarrow \psi(x), \psi_{l} \rightarrow \psi\left(x_{0}\right)$ and $(n-l) \Delta \rightarrow x-x_{0}$ we see that 


$$
\left(\psi_{l}-\psi_{l-1}\right) / \Delta
$$

must also approach a limit. Denoting this limit by $\alpha\left(x_{0}\right)$ we have from (4.6)

$$
\frac{\psi(x)}{2}-\frac{\psi\left(x_{0}\right)}{2}-\frac{\left(x-x_{0}\right)}{2} \alpha\left(x_{0}\right)=\int_{x_{0}}^{x} \int_{x_{0}}^{\xi}(s+u V(x)) \psi(x) d x d \xi
$$

Since (4.7) holds for all $x$ and $x_{0}$ which are both less than 0 or both greater than 0 we infer that for $x \neq 0$

$$
\frac{1}{2} \frac{d^{2} \psi}{d x^{2}}-(s+u V(x)) \psi=0, \quad x \neq 0 .
$$

It also follows that

$$
\alpha(x)=d \psi / d x, \quad x \neq 0 .
$$

Coming back to the difference equation (3.6) we get, for $l<-1$ and $n>1$, by summation

$$
\frac{1}{2} \frac{\psi_{n+1}-\psi_{n}}{\Delta}-\frac{1}{2} \frac{\psi_{l}-\psi_{l-1}}{\Delta}=\Delta \sum_{m=l}^{n} \frac{\exp \left\{\left(s+u V_{m}\right) \tau\right\}-1}{\tau} \psi_{m}-\exp (s \tau) \text {. }
$$

In the limit, as $n \Delta \rightarrow x>0$ and $l \Delta \rightarrow x_{0}<0$, we obtain

$$
\frac{1}{2} \frac{d \psi}{d x}-\frac{1}{2} \frac{d \psi}{d x_{x=x_{0}}}=\int_{x_{0}}^{x}(s+u V(x)) \psi(x) d x-1 .
$$

If we now let $x \rightarrow 0$ from the right and $x_{0} \rightarrow 0$ from the left we are led to the equality

$$
\psi^{\prime}(+0)-\psi^{\prime}(-0)=-2,
$$

which shows that $\psi(x)$ is indeed a fundamental solution of (4.8).

We have already seen that

$$
\psi_{m}<\frac{\tau}{2 \pi \Delta} \int_{0}^{2 \pi} \frac{\cos m \xi \cos \xi}{1-\cos \xi \exp (-s \tau)} d \xi
$$

and since it is easy to show that, as $\Delta \rightarrow 0, m \Delta \rightarrow x$, the right-hand side of (4.11) approaches $(2 s)^{-1 / 2} \exp \left\{-(2 s)^{1 / 2}|x|\right\}\left({ }^{4}\right)$ we obtain

$$
\psi(x) \leqq(2 s)^{-1 / 2} \exp \left\{-(2 s)^{1 / 2}|x|\right\}
$$

and, in particular,

$$
\psi(x) \rightarrow 0, \quad x \rightarrow \pm \infty .
$$

( $\left.{ }^{4}\right)$ Write the right-hand side of (4.11) in the form $(\tau / \pi \Delta) \int_{0}^{\pi}(\cos m \xi \cos \xi /(1-\cos \xi \exp$ $(-s \tau)) d \xi$ and make the substitution $\xi=\eta \Delta$. We then recognize almost immediately that the integral approaches $(2 / \pi) \int_{0}^{\infty}\left(\cos x \eta /\left(2 s+\eta^{2}\right)\right) d \eta=(2 s)^{-1 / 2} \exp \left\{-(2 s)^{1 / 2}|x|\right\}$. 
It is also easy to show that

$$
\left|\frac{d \psi}{d x}\right| \leqq 2 .
$$

In fact, starting with the difference equation (3.6) and summing over $m$ from $-\infty$ to $\infty$ we obtain

$$
\Delta \sum_{m} \frac{\exp \left\{\left(s+u V_{m}\right) \tau\right\}-1}{\tau} \psi_{m}=\exp (s \tau) .
$$

Summing over $m$ from $-\infty$ to $l<-1$ we get

$$
\frac{1}{2} \frac{\psi_{l+1}-\psi_{l}}{\Delta}=\Delta \sum_{m \leqq l} \frac{\exp \left\{\left(s+u V_{m}\right) \tau\right\}-1}{\tau} \psi_{m}<\exp (s \tau)
$$

and hence, in the limit $\Delta \rightarrow 0, l \Delta \rightarrow x<0$,

$$
0 \leqq \frac{d \psi}{d x} \leqq 2,
$$

Likewise we prove that

$$
0 \leqq-\frac{d \psi}{d x} \leqq 2,
$$$$
x>0 \text {. }
$$

Conditions (4.13) and (4.14) imply trivially $\left(^{5}\right)$ that our fundamental solution is unique. Much less would insure uniqueness but the corresponding theorem would be less elementary.

Finally, we must show that

$$
\lim _{\Delta \rightarrow 0} \Delta \sum_{m} \psi_{m}=\int_{-\infty}^{\infty} \psi(x) d x
$$

This, however, follows easily from the estimates (4.11) and (4.12) and from the fact $\psi_{m} \rightarrow \psi(x)(m \Delta \rightarrow x)$, uniformly in every finite interval.

Actually, the use of the estimate (4.11) can be circumvented. We have

$$
\begin{aligned}
\Delta \sum_{|m \Delta|>a} \psi_{m} & =\sum_{|m \Delta|>a} \chi_{m}=\tau \sum_{|m \Delta|>a} \sum_{k=1}^{\infty} \phi(m \Delta, k \tau) \exp (-(k-1) s \tau) \\
& =\tau \sum_{k=1}^{\infty} \exp (-(k-1) s \tau) \sum_{|m \Delta|>a} \phi(m \Delta, k \tau)
\end{aligned}
$$

(5) Assuming the existence of two fundamental solutions $\psi_{1}$ and $\psi_{2}$ we put $\phi=\psi_{1}-\psi_{2}$ and note that $\phi$ satisfies the differential equation (4.8) and the conditions $\phi \rightarrow 0$ as $x \rightarrow \pm \infty,\left|\phi^{\prime}\right|<M$. Moreover, $\phi^{\prime}(+0)-\phi^{\prime}(-0)=0$. Multiplying the differential equation $\phi^{\prime \prime} / 2-(s+u V(x) \phi=0$ by $\phi^{\prime}$ and integrating between $-A$ and $B(A>0, B>0)$ we conclude that $\phi \equiv 0$ by the familiar trick of integrating by parts and letting $A$ and $B$ approach $+\infty$. 
and

$$
\sum_{|m \Delta|>a} \phi(m \Delta, k \tau) \leqq \text { Prob. }\left\{\left|X_{1}+\cdots+X_{k}\right|>\frac{a}{\Delta}\right\} .
$$

Using Tchebysheff's inequality we get

$$
\sum_{|m \Delta|>a} \phi(m \Delta, k \tau) \leqq \frac{k \Delta^{2}}{a^{2}}=\frac{k \tau}{a^{2}}
$$

and, finally,

$$
\begin{aligned}
\Delta \sum_{|m \Delta|>a} \psi_{m} & \leqq \frac{\tau^{2}}{a^{2}} \sum_{k=1}^{\infty} k \exp (-(k-1) s \tau) \\
& =\frac{1}{a^{2}}\left(\frac{\tau}{1-\exp (-s \tau)}\right)^{2}<\frac{E(s)}{a^{2}},
\end{aligned}
$$

where $E(s)$ depends on $s$ alone. Estimate (4.16) together with (4.12) implies (4.15). If we now recall (2.12) and (3.7) we can write in view of (4.15)

$$
g(u ; s)=\int_{-\infty}^{\infty} \psi(x) d x .
$$

Let us also remark (following Courant, Friedrichs and Lewy [5]) that the uniqueness of $\psi(x)$ implies that the sequence $\psi_{\Delta}(x)$ itself, and not merely a subsequence of it, converges to $\psi(x)$.

5. Inversion and examples. To find $\sigma(\alpha ; t)$ one must perform a double inversion. The inversion with respect to $s$ is usually the easier of the two. The following remark may sometimes prove useful. If one tries to find the fundamental solution of the partial differential equation

$$
\frac{\partial \omega}{\partial t}=\frac{1}{2} \frac{\partial^{2} \omega}{\partial x^{2}}-u V(x) \omega
$$

by the use of the Laplace transform, then one is led formally to the differential equation

$$
\frac{1}{2} \frac{d^{2} \psi}{d x^{2}}-(s+u V(x)) \psi=0
$$

$x \neq 0$.

On formal grounds one might, therefore, expect that the inversion with respect to $s$ will yield the fundamental solution (which becomes singular at $x=0$ for $t \rightarrow 0$ ) of (5.1). We do not pursue this connection in a general and rigorous manner because we feel that not enough would be gained by so doing. In fact, in most cases, the treatment of (5.1) is anyway reduced to a treatment of a corresponding Sturm-Liouville problem, that is, essentially to (5.2). 
We now illustrate the general theory on two examples which were treated previously by somewhat different methods.

EXAMPle 1. $V(x)=x^{2}$ (see [1] and [6]).

We must find the fundamental solution. of

$$
\frac{d^{2} \psi}{d x^{2}}-\left(2 s+2 u x^{2}\right) \psi=0
$$

Consider first the corresponding eigenvalue problem

$$
\frac{d^{2} \psi}{d x^{2}}-2 u x^{2} \psi=\lambda \psi
$$

subject to the condition $\psi \rightarrow 0$, as $x \rightarrow \pm \infty$. Setting

$$
\psi=D(x / \alpha)
$$

we obtain

$$
D^{\prime \prime}\left(\frac{x}{\alpha}\right)+\left(-\lambda \alpha^{2}-2 u \alpha^{4}\left(\frac{x}{\alpha}\right)^{2}\right) D\left(\frac{x}{\alpha}\right)=0 .
$$

Setting

$$
\alpha^{2}=(2 u)^{-1 / 2} / 2
$$

we obtain

$$
D^{\prime \prime}\left(\frac{x}{\alpha}\right)+\left(-\frac{\lambda}{2}(2 u)^{-1 / 2}-\frac{1}{4}\left(\frac{x}{\alpha}\right)^{2}\right) D\left(\frac{x}{\alpha}\right)=0
$$

which we recognize as Weber's equation. It is well known that in order to have solutions which vanish at $\pm \infty$ one must have

$$
-\frac{\lambda}{2}(2 u)^{-1 / 2}=n+\frac{1}{2}
$$

where $n=0,1,2, \cdots$.

The corresponding eigenfunctions are Hermite functions and one has

$$
\int_{-\infty}^{\infty} D_{m}\left(\frac{x}{\alpha}\right) D_{n}\left(\frac{x}{\alpha}\right) d x=\alpha(2 \pi)^{1 / 2} n ! \delta(m, n)
$$

The fundamental solution we seek is given by the formula

$$
\psi(x)=\frac{(2 \pi)^{-1 / 2}}{2 \alpha} \sum_{n=0}^{\infty} \frac{D_{n}(x / \alpha) D_{n}(0)}{n !\left[s+(n+1 / 2)(2 u)^{1 / 2}\right]} \cdot
$$

Noticing that 


$$
\frac{1}{s+(n+1 / 2)(2 u)^{1 / 2}}=\int_{0}^{\infty} \exp (-s t) \exp \left\{-(n+1 / 2)(2 u)^{1 / 2} t\right\} d t
$$

and using the formula

$$
\begin{aligned}
& \sum_{n=0}^{\infty} \frac{D_{n}(\xi) D_{n}(\eta)}{n !} \exp (-n \tau) \\
& \quad=(1-\exp (-2 \tau))^{-1 / 2} \exp \left(\frac{\xi^{2}+\eta^{2}}{4}\right) \exp \left\{-\frac{\xi^{2}+\eta^{2}-2 \xi \eta \exp (-\tau)}{2(1-\exp (-2 \tau))}\right\}
\end{aligned}
$$

we obtain

$$
\begin{aligned}
\psi(x)= & \frac{(2 \pi)^{-1 / 2}}{2 \alpha} \exp \left(\frac{x^{2}}{4 \alpha^{2}}\right) \int_{0}^{\infty} \exp (-s t) \\
& \cdot \exp \left(-\frac{(2 u)^{1 / 2}}{2} t\right)\left(1-\exp \left(-2(2 u)^{1 / 2} t\right)\right)^{-1 / 2} \\
& \cdot \exp \left\{-\frac{x^{2}}{2 \alpha\left(1-\exp \left((2 u)^{1 / 2} t\right)\right)}\right\} d t
\end{aligned}
$$

An easy calculation gives

$$
\int_{-\infty}^{\infty} \psi(x) d x=\int_{0}^{\infty} \exp (-s t)\left(\operatorname{sech}(2 u)^{1 / 2} t\right)^{1 / 2} d t
$$

or

$$
f(u ; t)=\left(\operatorname{sech}(2 u)^{1 / 2} t\right)^{1 / 2} .
$$

Some of the above calculations were carried out in a formal manner but they can be easily justified.

Example 2. $V(x)=(1+\operatorname{sign} x) / 2($ see [3]).

Equation (5.2) for $x>0$ becomes

$$
\frac{1}{2} \frac{d^{2} \psi}{d x^{2}}-(s+u) \psi=0
$$

and for $x<0$

$$
\frac{1}{2} \frac{d^{2} \psi}{d x^{2}}-s \psi=0
$$

Thus (since $\psi$ must vanish at $\pm \infty$ )

$$
\begin{array}{ll}
\psi(x)=A \exp \left\{-(2 s+2 u)^{1 / 2} x\right\}, & x>0 \\
\psi(x)=B \exp \left\{(2 s)^{1 / 2} x\right\}, & x<0 .
\end{array}
$$

Continuity at $x=0$ yields $A=B$ and the condition 


$$
\psi^{\prime}(+0)-\psi^{\prime}(-0)=-2
$$

implies

$$
A=\frac{2^{1 / 2}}{s^{1 / 2}+(s+u)^{1 / 2}} .
$$

Thus, after a few minor transformations

$$
\int_{-\infty}^{\infty} \psi(x) d x=s^{-1 / 2}(s+u)^{-1 / 2}
$$

For sufficiently large $s$

$$
s^{-1 / 2}(s+u)^{-1 / 2}=\sum_{k=0}^{\infty}\left(\begin{array}{c}
-1 / 2 \\
k
\end{array}\right) \frac{u^{k}}{s^{k+1}}
$$

and hence, inverting with respect to $s$,

$$
f(u ; t)=\sum_{k=0}^{\infty} \frac{1}{k !}\left(\begin{array}{c}
-1 / 2 \\
k
\end{array}\right)(u t)^{k}=\frac{2}{\pi} \int_{0}^{\pi / 2} \exp \left(-u t \cos ^{2} \theta\right) d \theta .
$$

Recalling that

$$
f(u ; t)=\int_{0}^{\infty} \exp (-\alpha u) d_{\alpha} \sigma(\alpha ; t)
$$

we see that $\sigma(\alpha ; t)$ is the distribution function of $t \cos ^{2} \theta$ in $0 \leqq \theta \leqq \pi / 2$.

Finally,

$$
\sigma(\alpha ; t)=\frac{2}{\pi} \arcsin \left(\frac{\alpha}{t}\right)^{1 / 2}, \quad 0 \leqq \alpha \leqq t .
$$

\section{BibLIOGRAPHY}

1. P. Erdös and M. Kac, On certain limit theorems of the theory of probability, Bull. Amer. Math. Soc. vol. 52 (1946) pp. 292-302.

2. M. Kac, On the average of a certain Wiener functional and a related limit theorem in calculus of probability, Trans. Amer. Math. Soc. vol. 59 (1946) pp. 401-414.

3. P. Erdös and M. Kac, On the number of positive sums of independent random variables, Bull. Amer. Math. Soc. vol. 53 (1947) pp. 1011-1020.

4. R. Fortet, Quelques travaux recent sur le mouvement Brownien, to appear in Annales de l'Institut Henri Poincaré.

5. R. Courant, K. Friedrichs and H. Lewy, Über die partiellen Differenzengleichungen der mathematischen Physik, Math. Ann. vol. 100 (1928) pp. 32-74.

6. R. H. Cameron and W. T. Martin, The Wiener measure of Hilbert neighborhoods in the space of real continuous functions, Journal of Mathematics and Physics vol. 23 (1944) pp. 195209.

CORNEll UNIVERSity, ITHACA, N. Y. 